\title{
Ajuste psicológico emocional en la población infanto juvenil en situación de conflicto social, una aproximación a los estilos de afrontamiento personal. El caso de Navarra* The emotional and psychological adjustment of pubertal children who live in social conflict situation, an approach on personal confrontation styles. The Navarra's case 0 ajuste psicológico emocional na população infanto-juvenil em situação de conflito social, uma aproximação aos estilos de enfrentamento pessoal. 0 caso de Navarra
}

Miguel Ángel Gurbindo Marin ${ }^{1}$

Universidad Pública de Navarra, España

\begin{abstract}
Resumen
La infancia y adolescencia en situación de riesgo/conflicto social configura una realidad que por sus características da lugar a una de las múltiples caras del espacio social de la exclusión. Constituye un colectivo que aunque en términos cuantitativos no sea en la actualidad y en el contexto navarro, muy grande, si que lo es su situación de desprotección, deterioro psicosocial y conflicto social permanente. En nuestro caso hablamos de un colectivo cuyas señas de identidad se han ido creando en contextos de desprotección severa y prolongada, sin una cobertura básica de sus necesidades físico-biológicas, emocionales y sociales, así como con un insuficiente grado de competencias y habilidades para la interacción socio-familiar. Todo lo cual, hace que este colectivo, esté cada vez más presente en el ámbito de actuación de la salud mental infanto-juvenil.
\end{abstract}

Palabras clave: Protección, Infancia, Riesgo, Adolescencia, Crisis, Exclusión social, Salud mental.

\section{Abstract}

This paper focuses on analysis of children and adolescents who live in social conflict. Their identity has been formed in a severe and prolonged context of appalling neglect which does not cover their physical-biological, emotional and social needs. Therefore those human beings do not develop their skills to interact with their relatives and ensure the stability of the family unit. The extent of this phenomenon, as one of the many faces of social exclusion, is not so big in Navarra; however it must be taken into account that lack of protection, psychosocial impairment and permanent social conflict increases the risk that pubertal children suffer mental health problems.

Key words: Protection, Childhood, Risk, Adolescence crisis, Social exclusion, Mental health.

Resumo

A infância e a adolescência em situação de risco / conflito social configura uma realidade que por sua natureza leva a uma das muitas faces de espaço de exclusão social. É um coletivo que, embora quantitativamente não seja grande atualmente no contexto Navarro, a sua falta de proteção sim é grande, assim como a deterioração psicossocial e o conflito social permanente. No nosso caso, falamos de um grupo cuja identidade foi sendo criado no contexto de falta de proteção, sem uma cobertura básica de suas necessidades físicas, biológicas, emocionais e sociais, bem como um grau insuficiente de habilidades de competência para a interação social e familiar. Tudo isso faz com que este grupo esteja cada vez mais presente no âmbito da atuação da saúde mental infanto-juvenil.

Palavras-chave: Proteção, Infância, Risco, Crise da adolescência, Exclusão social, Saúde mental.

Cómo referenciar este artículo: Gurbindo, M. (2016). Ajuste psicológico emocional en la población infanto juvenil en situación de conflicto social, una aproximación a los estilos de afrontamiento personal. El caso de Navarra. Pensamiento Americano, 9(16), 58-77.

\section{c) $\boldsymbol{\theta} \otimes \Theta$}

Recibido: Julio 31 de 2015 • Aceptado: Octubre 23 de 2015

El presente artículo está vinculado al proyecto de investigación longitudinal desarrollado en Navarra a lo largo del periodo 20072014: "Adolescencia en riesgo social. Un estudio de las situaciones de conflicto social severo y prolongado en la adolescencia, abordadas desde el Sistema de Protección a la Infancia en Navarra” Gurbindo, M. (2014).

1. Profesor asociado en el Departamento de Trabajo Social de la Universidad Publica de Navarra. Técnico de valoración situaciones de desprotección en la infancia. Gobierno de Navarra. Doctor por la Universidad Pública de Navarra. m.a.gurbindo@terra.com 
"Emotional and psychological adjustment of children and young minors living within social conflict. An approach to personal confrontation styles. The case of Navarre".

\section{Introducción}

La conducta antisocial y/o la conducta desadaptada en tanto que el exponente principal de las situaciones de conflicto social en la adolescencia, no tiene su génesis o razón de ser en un solo factor o hecho determinante. El conflicto social adolescente y la conducta desadaptada, extienden sus raíces en un conjunto de factores complejos y multidimensionales de carácter personal, familiar, escolar, sanitario y/o social. Factores, todos ellos construidos desde la interacción continua y bidireccional del individuo con los sistemas del ambiente. Desde esta perspectiva y en coherencia con el modelo ecosistémico, entendemos al individuo en desarrollo (en nuestro caso el/la adolescente), como el resultado de una serie de transiciones vitales y transacciones sociales, a través de las cuales busca acomodarse en un contexto en permanente cambio.

Las habilidades y competencias personales en la búsqueda de dicha acomodación, las oportunidades vitales con las que se haya podido contar, las exigencias establecidas desde el entorno natural de convivencia, así como la cognición que de todo ello se haga, forman parte ineludible a la hora de poder analizar el origen de la disfuncionalidad en las interacciones con el medio.
En este artículo nos acercaremos a las situaciones de conflicto social en la adolescencia desde el análisis de algunas de las características personales y cognitivas, así como de sus efectos en la salud mental infanto-juvenil de los/as menores implicados. En este sentido, centraremos el análisis en aquellos aspectos y características psicosociales que acaban determinando en los/as menores: una mayor o menor conciencia del problema, un determinado modelo de atribución (externa o interna) al patrón instaurado de conducta, así como los propios estilos y estrategias de afrontamiento personal ante las situaciones adversas o estresantes. Es decir, todos aquellos aspectos, estrategias y maneras de actuar que en gran medida configuran las señas de identidad del ámbito personal, cognitivo y de salud mental de los/as menores atendidos en el Sistema de Protección a la Infancia (SPI).

¿Pero de qué estamos hablando cuando nos referimos al concepto de conflicto social severo y prolongado en la adolescencia? Desde una primera aproximación y de acuerdo a lo recogido en la Ley Foral 15/2005 de Promoción, Atención y Protección a la infancia en Navarra: "se consideran menores en conflicto social, encuadrados en el sistema de protección, aquéllos que, por su situación de grave inadaptación, pudieran encontrarse en riesgo de causar perjuicios a sí mismos o a otras personas" (Gobierno de Navarra, 2005 p.57).

Evidentemente, la realidad diaria de las si- 
tuaciones sobre las que hay que intervenir en el contexto del SPI, supera ampliamente el recorrido de esta definición. No obstante, somos conscientes de las dificultades de establecer una nueva aproximación al concepto del conflicto social severo y prolongado en la adolescencia, que vaya más allá del reduccionismo de asociarlo, únicamente, a las manifestaciones conductuales. Pese a ello, y de acuerdo a la línea argumental mantenida en el marco teórico que da sentido a este artículo, proponemos una nueva aproximación conceptual, cuyas señas de identidad estén sustentadas en el carácter complejo y multidimensional del mismo. Decimos complejo en tanto que, en su propia génesis y en su desarrollo, hay una diversidad de variables, así como de elementos interrelacionados, que rompen las rutinas y expectativas ordinarias de la vida cotidiana. Multidimensional, en la medida que está transversalizado por una relación continua y bidireccional entre el individuo y las diferentes estructuras o sistemas de socialización primaria y secundaria. Es decir, estamos ante una tipología de conflicto social con señas de identidad propias y diferenciadas de otro tipo de conflictos, en tanto que: 1 . no es un conflicto exclusivamente con adultos; 2 . no va dirigido (al menos conscientemente) al cambio social o enfrentamiento con el sistema o las instituciones de control; 3. no se expresa colectivamente, sino de forma individual; 4. no suele estar focalizado en un solo ámbito, sino que, habitualmente, tiende a la generalización de la conducta hacia los diferentes contextos o sistemas de socialización por donde el/la menor transita e interactúa; y 5. tiene en su expresión un alto componente de desajuste emocional, manifestado mediante la búsqueda de la conducta autodestructiva.

Estamos hablando de un tipo de conflicto que hunde sus raíces en lo personal, en los vínculos familiares y las relaciones de apego disfuncionales. En la exposición a la violencia, en las transiciones vitales y en las transacciones sociales. En la desprotección a la infancia, así como en la inadaptación a las nuevas realidades sociales, marcadas por el cambio y la nueva doctrina de la flexibilidad. Las situaciones a las que nos referimos, configuran una forma de conflicto social, en definitiva, deslocalizada de lo grupal a lo individual, que se expresa en el marco de las relaciones personales, familiares, afectivo-sexuales, formativo-laborales y judiciales. Todo ello, desde un nuevo patrón conductual, marcado unas veces por lo disruptivo y lo disocial, la violación de las normas sociales, o los trastornos del comportamiento; otras, por la inhibición, el mutismo y la incomunicación; $y$, finalmente, otras, por la búsqueda autodestructiva, expresada mediante las prácticas de riesgo incontrolado, las conductas autolíticas, o la desconexión social.

Se trata de un sector de la población adolescente y/o joven situado en conflicto social severo y prolongado, cuya causalidad está sustentada en un contexto de interacción continua y bidireccional con sus ámbitos referenciales de socialización: 
- Personal

- Familiar

- Social

- Escolar

- Laboral

- Judicial

Ámbitos referenciales, todos ellos, que, en la mayor parte de los casos, propician y se convierten en factores de riesgo favorecedores de convivencias, procesos, conductas y aprendizajes disfuncionales, que, a su vez y como resultado, dan lugar a procesos de socialización patológicos, en tanto que tienen lugar en: Contextos de convivencia familiar altamente disfuncionales, procesos de integración formativo-laboral altamente deficitarios, así como serios problemas en los procesos de socialización primaria y secundaria

Dada la amplitud y heterogeneidad de los contextos en los que a día de hoy se mueve el ejercicio profesional del Trabajo Social en infancia y adolescencia en riesgo, hemos delimitado el espacio de análisis a todos/as aquellos/as menores cuya situación de conflicto social severo y prolongado ha hecho inviable el mantenimiento de la convivencia en su unidad familiar de referencia, siendo necesario el establecimiento de medidas extremas de protección, como sin duda lo son la gestión de la guarda por parte de la Entidad Pública, esto es, de manera provisional o definitiva mediante el acogimiento residencial administrativo o en su caso judicial.
Para ello, hemos tomado como fuente de documentación y base para el análisis, los datos obtenidos en la investigación longitudinal ${ }^{1}$ que hemos llevado a cabo a lo largo del periodo 2007-2014, esto es, con el $100 \%$ (857), de los(as) menores atendidos en el SPI de Navarra a causa de su situación de conflicto social severa y prolongada.

\section{Objeto de estudio}

La investigación que ha dado pie al presente artículo responde al: "análisis de los factores de riesgo-protección implicados en el ajuste psicológico-emocional de la población infanto-juvenil en situación de conflicto social severo y prolongado". Esto es, dentro del espacio social y territorial de la Comunidad Foral de Navarra y de acuerdo al marco de actuación que establece el $\mathrm{SPI}^{2}$.

\section{Metodología}

La investigación llevada a cabo y que sirve de base para este artículo ha tenido un carácter descriptivo. Para ello, a la hora de definir el método de investigación, hemos tenido en cuenta: 1. la necesidad de darle un carácter exploratorio (dado el escaso desarrollo del objeto de investigación); 2. que los resultados de la

1. "Adolescencia en riesgo social. Un estudio de las situaciones de conflicto social severo y prolongado en la adolescencia, abordadas desde el Sistema de Protección a la Infancia en Navarra". Gurbindo, M. (2014).

2. El sistema de protección a la infancia en Navarra constituye un conjunto de servicios, programas, recursos y prestaciones dirigidas a detectar, notificar, investigar, valorar y en su caso intervenir en todos aquellos contextos en los que se estén dando situaciones de desprotección y/o conflicto social en los niveles de: riesgo de desprotección leve, moderada o grave y las situaciones de desamparo (G.N., 2002). 
misma tuvieran un carácter diagnóstico a fin de poder extraer generalizaciones significativas que contribuyan a un mayor conocimiento del fenómeno objeto de investigación; 3. que nos permitiera una estrategia de indagación intensiva sobre múltiples unidades de análisis (en nuestro caso, 858), sobre las que poder establecer un análisis descriptivo y correlacional que ponga énfasis en el análisis de las causas que propician el comportamiento de los individuos y sus circunstancias; y 4. que pudiera ser llevado a cabo desde una perspectiva inductiva, procesual y sistemática, así cómo, con una dimensión temporal de investigación con un carácter longitudinal o diacrónica. Todo lo cual, ha hecho que nos planteáramos llevar a cabo la presente investigación, tomando como referencia el método de "estudio de caso" 3 . Una metodología establecida desde el doble paradigma cualitativo-cuantitativo, llevada a cabo en dos fases y estrategias diferenciadas con una secuencia de aplicación temporal. De tal manera, que en una primera fase y mediante el uso de metodología cualitativa, se ha establecido el proceso de recogida y contraste de información. Esto es, mediante las técnicas de la entrevista en profundidad con carácter semiestructurada, la observación participante y el análisis documental. La segunda fase, de carácter cuantitativo, ha tenido por objetivo la clasificación, codificación, operacionalización de variables e indicadores científicamente validados mediante las baterías de test recogidos

3. El estudio de caso es definido como el examen de un fenómeno en específico, tales como un programa, un evento, una persona, un proceso, una institución o un grupo social. en García Mediavilla (1989) y Millon (1999), así como a partir del análisis estadístico de los datos obtenidos, ya sean estos de carácter primario o secundario. El tratamiento y análisis de los datos recogidos se ha llevado a cabo mediante el paquete estadístico SPSS. V.20 para Windows.

\section{Variables utilizadas}

El presente estudio se ha llevado a cabo mediante dos grupos diferenciados de variables: el primero de ellos con un carácter sociodemográfico o situacional recoge el sexo, la edad, la procedencia y el nivel de estudios. En segundo lugar hemos estudiado un grupo de variables asociadas directamente con el objeto de investigación, como son la autoestima y la autoconfianza, el grado de competencias en habilidades sociales, el estilo de afrontamiento personal, la conciencia del problema y motivación para el cambio, así como el nivel de resiliencia.
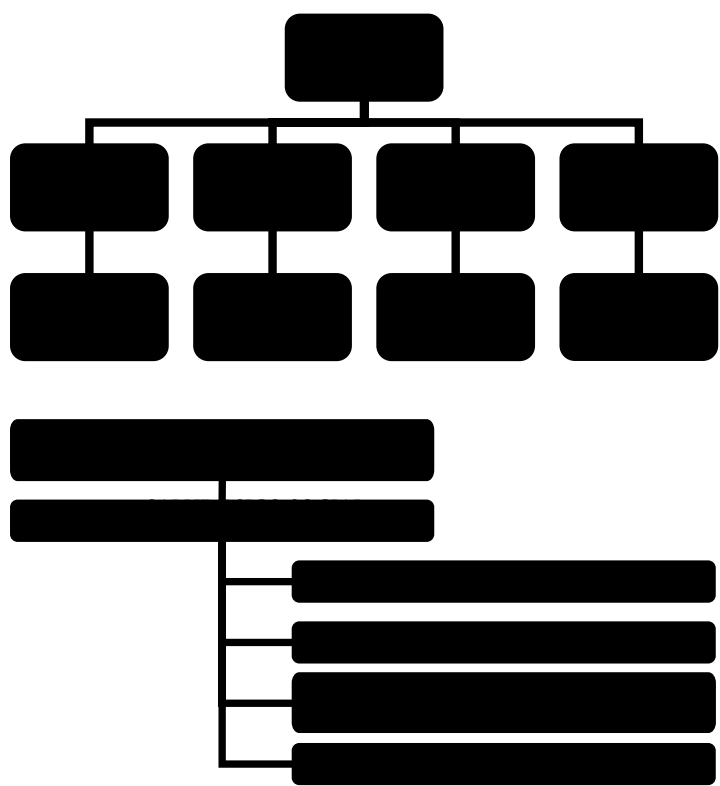


\section{Universo de estudio, muestra y ámbito geo-} gráfico de investigación

Formado por el conjunto de menores situados en el tramo de edad 12-19 años, atendidos por el SPI de Navarra por estar inmersos en una situación de conflicto social severo y prolongado. Menores sobre los que, a su vez, se esté llevando una intervención de acuerdo a los protocolos de detección, notificación y valoración establecidos en el Manual de Intervención en Situaciones de Desprotección del Gobierno de Navarra (Navarra, 2002, pp.30-57) .

Todo lo cual, hace que en términos cuantitativos y tomando como referencia la población acumulada en los últimos ocho años (periodo de investigación), estamos hablando de un universo 1200 menores, con una tasa media de incorporación de 150 menores por año. En cuanto a la muestra establecida para el desarrollo de esta investigación, está formada por 857 casos o conjunto de unidades de análisis, correspondientes al $100 \%$ de los/as menores ingresados en el Centro de Observación y Acogida ${ }^{5}$ a lo largo de los últimos ocho años (espacio temporal para la investigación).

4. El Manual de Intervención en Situaciones de Desprotección del Gobierno de Navarra, constituye una herramienta de trabajo en la que se establecen los protocolos para llevar a cabo las diferentes fases de intervención con menores en situación de riego social.

5 En adelante COA. Centro de primera acogida para situaciones de urgencia en régimen de institucionalización temporal (entre 2 y 3 meses), donde se llevan a cabo los procesos de valoración psicosocial en profundidad, el establecimiento del diagnóstico diferencial y gradiente de gravedad en cuanto a la situación de conflicto social, la orientación para el posterior proceso de intervención, así como el establecimiento de otras medidas complementarias de protección: medidas cautelares.
De tal manera que cada año se han tomado, como objeto de investigación, una media de 108 nuevas unidades de análisis (100\% de los(as) menores ingresados en COA). En este sentido, entendemos que la muestra es suficientemente representativa, en tanto que recoge en sí misma el $100 \%$ de los/as usuarios detectados, notificados e incorporados a lo largo del periodo 2007-2014 (ambos inclusive), por estar inmersos en situaciones de conflicto social severo y prolongado. La muestra es igualmente significativa, en la medida que todos/as sus integrantes han participado de un proceso de intervención protocolizada (proceso de acogida, proceso de investigación-valoración, establecimiento de diagnóstico diferencial, plan personalizado de intervención y seguimiento posterior a la intervención).

Por último, se ha tenido a la Comunidad Foral de Navarra como contexto geográfico, y el periodo 2007-2014 (ambos inclusive) como espacio temporal de investigación.

\section{Resultados obtenidos}

\section{Datos sociodemográficos}

Del conjunto de la población objeto de atención es de destacar la falta de diferencias significativas por razón de género, en la medida que el $51 \%$ de ellos son de signo masculino, frente a un $49 \%$ de signo femenino. Todo lo cual, nos lleva a pensar que la conducta desadaptada en tanto que primera manifestación de la situación de conflicto social en la adolescencia, responde a múltiples causas de 
naturaleza compleja y multidimensional. Entre otras, las características del contexto familiar, social y/o cultural, las transiciones vitales e interacciones con el medio, la deprivación afectiva, así como múltiples hechos, situaciones o circunstancias asociadas a itinerarios de exclusión social, y no tanto a diferencias derivadas del sexo. En este sentido, hemos podido ir comprobando cómo en los últimos 10 años se ha ido produciendo un incremento sostenido en el tiempo en cuanto a la presencia femenina, asociada fundamentalmente a conflictos derivados del área de convivencia familiar y socio-relacional. Dichos conflictos, se ponen de manifiesto mediante una dinámica conductual altamente desadaptada, expresada mediante fugas del domicilio familiar, graves enfrentamientos con las figuras parentales de referencia, relaciones sexuales altamente inadecuadas y/o de riesgo, consumos elevados de sustancias tóxicas, pertenencia a grupos/bandas de carácter predelictivo, así como pequeños hurtos, en los que habitualmente no hay empleo de violencia.

Contrariamente a lo que cabría esperar, el estudio de la variable procedencia en relación a los/as menores atendidos, establece una ligera prevalencia tanto en términos absolutos como porcentuales de la población de origen extranjera respecto de la población nativa: 447 menores de origen extranjero (52\%) frente 411 de origen nativo (48\%). El análisis en términos absolutos, apenas si es significativo, dado que únicamente refleja una diferencia de 36 menores en un periodo de ocho años. No obstante, si tomamos como referencia los valores relativos en cuanto a la incidencia o peso sobre sus poblaciones de origen, si que hay motivos de alarma, esto es, dado el alto grado de sobrerrepresentación entre los usuarios de origen extranjero, comparativamente con sus iguales nativos. Mientras los/as usuarios nativos constituyen un $0,5 \%$ respecto de su cohorte poblacional (12-19 años), los/as usuarios de origen extranjero, suponen con respecto a la suya, un $4 \%$, lo cual representa un porcentaje ocho veces mayor que el que debería corresponderles.

En cuanto a la media de edad de los menores atendidos en el momento de entrada en el COA, está situada en los 15,61 años, con un bajo nivel de dispersión (desviación típica $\boldsymbol{\sigma} 1$, $41)$, y sin apenas cambios significativos por razones de género o procedencia. Es de reseñar cómo el $85 \%$ de la población objeto de estudio está agrupada en torno a los intervalos 14-15 años (41 \%) y 16-17 años (44\%), periodo definido por la OMS como el de la adolescencia media, y espacio temporal en el que se desarrollan una parte importante de los conflictos personales asociados a la configuración de la identidad personal y grupal. Así mismo constituye el tiempo de gran parte de las transiciones vitales de carácter normativo, como son: la entrada en la edad penal de menores, el fin de la obligatoriedad de la enseñanza académica, el inicio de la vida laboral, el ejercicio del derecho a la autonomía sanitaria y el acceso a la emancipación. La media de edad a la salida del SPI está situada en los 17,75 años, lo cual nos ha dado como media de estancia en el SPI, 2,13 años por menor. 
En cuanto a la tipología y estructura familiar, el primer dato a destacar de los datos obtenidos, es que el $47 \%$ de las familias objeto de atención corresponde a modelos con ausencia de alguna de las figuras parentales de referencia (familias monoparentales), frente a un $39 \%$ de los usuarios procedentes de familias con presencia de dos figuras adultas con funciones de parentalidad (familia nuclear o tradicional + familia reconstituida). En este sentido, las familias monoparentales constituyen, en el contexto de nuestra investigación, el modelo familiar más prevalente, llamando la atención su elevada sobrerrepresentación; más aun, si lo comparamos con su grado de representación en el conjunto de la población Navarra, donde la tasa de incidencia era, para el año 2007, en torno al $4 \%$ (casi nueve veces menor), o la establecida a nivel nacional en la que la tasa de monoparentalidad simple apenas alcanza el $6 \%$ (Instituto Navarro de Estadística, 2007, p.87).

La tipología de familia tradicional, nuclear o heterosexual, establecida en primeras nupcias y en cuyo seno están claramente diferenciados los roles, en la población objeto de estudio, deja de ser hegemónica, ya que cómo hemos podido ver apenas tiene una incidencia global de un $39 \%$. La familia con carácter reconstituida ostenta una incidencia dentro de la población objeto de análisis, en torno al $10 \%$. Por último y con un $4 \%$ de incidencia, aparecen un grupo de menores cuya estructura familiar ha perdido sus dos figuras parentales de referencia, asumiendo ese papel, las familias extensas con funciones de parentalidad, las cuales se confi- guran con base en procesos de acogimiento familiar. Dentro de este grupo, aparecen aquellas situaciones en las que se han perdido las referencias familiares de origen debido a contextos de transnacionacionalidad, desamparo y/o abandono. Estas, corresponden a situaciones de institucionalización del menor debido a: 1 . Ruptura familiar debida a malos tratos, ingreso en prisión de las figuras parentales, abandono y/o fracaso de las medidas de acogimiento familiar; y 2. Situaciones derivadas de los procesos migratorios (fundamentalmente población del norte de África), en las que algunos menores se trasladan por su cuenta sin contar con un núcleo o red familiar de acogida. En estos casos, la administración acaba cumpliendo, de forma subsidiaria y hasta la mayoría de edad, las funciones tradicionales de la familia.

\section{Ajuste psicológico-emocional}

El sentido de este apartado no es otro que valorar el grado de ajuste psicológico emocional, o lo que es lo mismo, el grado de autoestima y autoconfianza, las experiencias de apego, así como las estrategias de afrontamiento personal con la aparición de dinámicas de conflicto social en la adolescencia. En este sentido hemos podido comprobar cómo las situaciones de conflicto social en la adolescencia tienen en su génesis y en la gran mayoría de los casos observados, indicadores de tipo cognitivo-emocional asociados: de una parte, a la propia estructura de personalidad, y de otra, a los diferentes acontecimientos que han ido configurando la trayectoria vital de los/as menores objeto de atención. Acontecimientos y situaciones que 
en muchos de los casos, se han mantenido cronificados en el tiempo, hasta ser percibidos como señas de identidad propias del sistema familiar. En palabras de Escudero (2013): "estaríamos hablando de experiencias altamente dolorosas, asociadas a la construcción del apego y el vínculo intrafamiliar. Sucesos en unos casos de carácter desprotector y/o maltratante difíciles de sobrellevar" (pp.110-125). En otros casos, a circunstancias vinculadas a sucesos estresantes de carácter no normativo surgidos en el tiempo de la adolescencia.

Todos estos hechos, situaciones o circunstancias vitales y la cognición que de todo ello han ido haciendo los/as menores, ha dado características propias a los estilos comunicacionales, los niveles de autoestima o a la propia percepción que se tenga del "otro/otra”. Así mismo, ha condicionado de manera evidente los estilos de afrontamiento personal, la mayor o menor capacidad para la tolerancia a la frustración, los niveles de impulsividad, o el grado de competencias adquiridas para la resolución pacífica de conflictos.

\section{Las experiencias de apego}

De los datos obtenidos en relación a las experiencias de apego, podemos decir que la relación de apego establecida entre el/la menor y sus progenitores constituye una característica esencial para poder entender las capacidades y disposiciones para el cambio. En este sentido y de acuerdo con Escudero (2013), el vínculo y la relación de apego adecuada, va a posibilitar el establecimiento de un soporte socializador adecuado a la edad y momento evolutivo del/ la menor (pp.110-125). Por el contrario, los modelos de apego disfuncionales, constituyen la base para el inicio de las conductas desadaptadas de agresividad, evitación y conflicto. Conductas, todas ellas, asociadas a una falta de seguridad en sí mismo/a, a una falta de autoestima y autoconfianza. En definitiva, a un déficit de capacidad para reconocer y gestionar de una forma adecuada las propias emociones.

Los menores faltos de vínculo y apego seguro, reproducen en sus interacciones con los/ adultos y el grupo de iguales, modelos relacionales disfuncionales, copiando y reproduciendo las conductas aprendidas de sus progenitores. En este sentido, hemos podido igualmente comprobar cómo la mayor o menor consistencia del vínculo que se haya ido construyendo a lo largo de la crianza entre las figuras parentales y los/as menores, incide de manera directa en la mayor o menor estabilidad psicológica-emocional de los/as mismos/as. De la misma manera y derivado de ello, influye en un mayor o menor grado de autoestima, autoconfianza, capacidad de resiliencia ${ }^{6}$, así como en la mayor o menor presencia de una amplia gama de problemas emocionales, incluyendo sintomatología ansioso-depresiva y/o trastornos del vínculo, del comportamiento o del estado de ánimo.

\footnotetext{
6. La resiliencia está definida como la capacidad para recuperarse y mantener una conducta adaptativa después del abandono o incapacidad inicial al iniciarse un evento estresante Barudi (2005).
} 
Tal como podemos ver en la Tabla 1 , los modelos de apego más prevalentes en la población objeto de atención tienen un carácter inseguro, desorganizado y/o ambivalente. Así mismo, señalar cómo el 74 \% de los/as menores, han tenido historias de crianza llevadas a cabo desde modelos de apego disfuncionales (apego inseguro/ambivalente/evitativo/ desorganizado).

Tabla 1. Distribución de menores según origen y tipología de apego

\begin{tabular}{|l|c|c|c|}
\hline $\begin{array}{c}\text { N=858 } \\
\text { Tipo de apego }\end{array}$ & $\begin{array}{c}\text { Población } \\
\text { origen nativo }\end{array}$ & $\begin{array}{c}\text { Población } \\
\text { origen } \\
\text { extranjero }\end{array}$ & Total \\
\hline Apego seguro & 101 & 122 & 223 \\
\hline $\begin{array}{l}\text { Apego inseguro } \\
\text { ambivalente }\end{array}$ & 310 & 325 & 635 \\
\hline TOTAL & 411 & 447 & 858 \\
\hline
\end{tabular}

Fuente: Elaboración propia a partir de los datos recogidos en "Adolescencia en riesgo social. Un estudio de las situaciones de conflicto social severo y prolongado en la adolescencia, abordadas desde el Sistema de Protección a la Infancia en Navarra" Gurbindo, M. (2014).

Los(as) menores socializados en estos entornos, responden fundamentalmente a dos contextos de crianza diferenciados: 1 . Los que proceden de familias altamente desestructuradas, con una trayectoria de negligencia, desatención, abandono y/o una larga exposición a la violencia; y 2. Los procedentes de procesos alargados en el tiempo en cuanto a deprivación afectiva y ausencia de sus figuras parentales. Este segundo grupo, en su mayoría de origen extranjero y procedente del área Latinoamericana, está caracterizado por: 1 . Un largo periodo de separación de la figura materna; 2 . Presencia de familia extensa con funciones de parentalidad; y 3. La vivencia en los menores de un doble sentimiento de abandono, el primero de ellos en la infancia, cuando la madre/ padre inicia el proyecto migratorio, dejándoles al cuidado del padre o de la familia extensa; el segundo, y coincidiendo con el proceso de reagrupamiento familiar, al tener que separarse de la que hasta entonces había sido su referencia familiar. En este contexto, los menores reagrupados deben reelaborar su relación de apego con la figura materna. Una figura desconocida, idealizada y ausente. Ausente durante los años de separación, y ausente de nuevo en el país de acogida, a causa de las condiciones laborales en las que habitualmente está inmersa. Los datos obtenidos en la investigación, coinciden con las investigaciones llevadas a cabo por otros autores: Barudy (2005) y Escudero (2013), en las cuales se establece una alta correlación entre los modelos de apego ${ }^{7}$ inseguro-desorganizado y la posterior aparición de patrones conductuales de carácter disocial, así como dificultades adaptativas en sus entornos relacionales. Por último y en cuanto al modelo ${ }^{8}$ de apego seguro presente en la población objeto de estudio (26\%), tal como muestra la Tabla 2, de los 223

7. A la hora de analizar la relación de apego, se ha tomado como referencia la clasificación presentada por Jorge Barudy en "los buenos tratos a la infancia" (Barudy, 2005).

8. Apego seguro: 1. Constituye una relación emocional perdurable con una persona en específico. 2. Dicha relación produce seguridad, sosiego, consuelo, agrado y placer. 3 . La pérdida o la amenaza de pérdida de la persona, evoca una intensa ansiedad. Se manifiesta en un tipo de relación donde la persona apegada siente una sensación de seguridad y confianza en la figura de apego, por lo tanto, le permite establecer vínculos con una persona a la cual él se acerca. Apego inseguro-desorganizado: se manifiesta en niños cuyo comportamiento trasluce mucha confusión, la madre y el padre son vividos desde la amenaza y la imprevisibilidad. Se da en entornos familiares muy disfuncionales, donde las figuras parentales sufrieron maltrato infantil. Se trata de familias en las que los niños/as han vivido casi toda clase de maltrato y violencia conyugal. 
casos en los que está presente, 56 corresponden a familias de minorías étnicas de origen nativo y extranjero (familias gitanas), 26 responden a población de origen extranjero denominada como Menas, 73 a familias de origen magrebí y subsahariana, 44 a población de origen nativa y de la Europa comunitaria, y finalmente 26 de ellas procede del área latinoamericana. En todos los casos, más allá del modelo familiar, el nivel de desprotección o el modelo educativo, el grado de apego seguro detectado, pone de manifiesto un fuerte vínculo entre los diferentes miembros de la unidad familiar, así cómo un nivel de factores de resiliencia superior al del resto de menores en los que hay una clara ausencia de apego seguro.

Tabla 2. Distribución de menores según origen y presencia de apego seguro

\begin{tabular}{|l|c|}
\hline \multicolumn{1}{|c|}{ Apego seguro } & N=223 \\
\hline Minorías étnicas O. Nativo y Europa Com. & 56 \\
\hline Menores no acompañados & 26 \\
\hline Origen magrebí-subsahariano & 73 \\
\hline Origen nativo & 44 \\
\hline Origen latino & 26 \\
\hline Total & 223 \\
\hline
\end{tabular}

Fuente: Elaboración propia a partir de los datos recogidos en "Adolescencia en riesgo social. Un estudio de las situaciones de conflicto social severo y prolongado en la adolescencia, abordadas desde el Sistema de Protección a la Infancia en Navarra" Gurbindo, M. (2014).

Las experiencias de apego inseguro en cualquiera de sus formas, los sucesos estresantes de carácter no normativo vividos en un contexto abandónico, maltratante o desprotector, van generando en los/as menores que las padecen, un profundo sentimiento de culpa. Escudero (2013) habla en este sentido, de una percepción negativa sobre sí mismos/as cuando la realidad de sus relaciones más cercanas y necesarias les dice que no han conseguido convertirse en la prioridad de sus padres. El grado de ansiedad e inseguridad generada en estos contextos de crianza, dan como resultado un déficit continuado de autoestima y autoconfianza (p.117).

\section{Autoestima y autoconfianza}

La autoestima y autoconfianza constituyen elementos indispensables para el establecimiento de relaciones interpersonales de calidad, así como para el desarrollo de competencias, habilidades y destrezas de gestión en la conducta emocional.

La seguridad en uno/a mismo/a, la autoconfianza generada en la historia de crianza, transfiere a los/as menores inteligencia emocional positiva, o lo que viene a ser lo mismo: capacidad para el afrontamiento de las conductas emocionales, capacidad para sentirse competente para la vida. El estado de fragilidad y confusión con el que el/la adolescente se enfrenta en la etapa adolescente a una cognición sobre sí mismo/a, hace que la autoestima ${ }^{9} \mathrm{y}$ autoconfianza ${ }^{10}$ sean elementos fundamentales en el desarrollo de la inteligencia emocional.

Lo observado en la investigación llevada a

9. "La autoestima hace referencia a la disposición a considerarse competente para hacer frente a los desafíos básicos de la vida y sentirse merecedor de felicidad" (Fulquez Castro, 2010, p.115).

10. La confianza en uno mismo es la parte del individuo que evalúa la eficacia de sus capacidades cuando se enfrenta o realiza cualquier tarea o actividad (Fulquez Castro, 2010, p.118). 
cabo hace que coincidamos con Fulquez Castro (2010) cuando plantea: "el hecho de valorarse y de creer en las propias capacidades hace que la persona se afronte a las situaciones porque considera que tiene capacidad de éxito y de resolución de conflictos"(p.124). Por el contrario, el/la adolescente con una baja autoestima no es capaz de sentirse competente para la vida, no es capaz de desarrollar habilidades sociales para la interacción, no tiene confianza en sí mismo/a y no es capaz de afrontar de forma positiva las experiencias negativas de la vida.

La población objeto de nuestro análisis, responde claramente a un perfil disfuncional en cuanto a niveles y calidad de autoestima y autoconfianza. Son menores, cuyas experiencias de apego han sido en su mayoría disfuncionales, con padres/madres ausentes, abandónicos, negligentes $y$, en muchos casos, maltratadores.

Los reportes recogidos en las entrevistas y la observación llevada a cabo en el COA, nos sitúa ante una población (ver Tabla 3), con un alto malestar interno, una imagen pobre de sí misma, un alto grado de culpabilización en cuanto al trato recibido por parte de sus figuras parentales, así como con grandes dificultades para modificar dicha situación. Constituye una población con serios problemas para vincular con las figuras adultas e inseguridad en las relaciones con iguales. Todo lo cual, crea importantes carencias en la capacidad para la regulación emocional, no pudiéndose evitar respuestas emocionales descontroladas en situaciones de inseguridad, ira, provocación o miedo. Así, se va configurando un patrón, en unos casos de desesperanza, inhibición e indefensión aprendida; en otros, de alta impulsividad, recelo y desconfianza en las relaciones, así como con un alto grado de agresividad, tanto en el plano físico como en el verbal.

Del análisis efectuado cabe señalar cómo, en el $77 \%$ de los/as menores están presentes (con diferentes niveles de intensidad), el total

Tabla 3. Distribución de menores según origen y características derivadas de un bajo nivel de autoestima y autoconfianza

\begin{tabular}{|c|c|c|c|c|c|}
\hline $\begin{array}{c}\mathrm{N}=\mathbf{8 5 8} \\
\text { Presencia de indicadores disfuncionales }\end{array}$ & Población O. nativo & Población 0. extranjero & Total & $\%$ & Media \\
\hline Desvalorización & 312 & 335 & 647 & $75 \%$ & \multirow{6}{*}{$77 \%$} \\
\hline Sentimiento de culpabilidad & 272 & 303 & 575 & $67 \%$ & \\
\hline Recelo/Desconfianza del adulto & 343 & 364 & 707 & $82 \%$ & \\
\hline $\begin{array}{l}\text { Dificultad para el establecimiento de vínculos } \\
\text { relacionales }\end{array}$ & 312 & 325 & 637 & $74 \%$ & \\
\hline Dificultad en las relaciones sociales & 348 & 364 & 712 & $83 \%$ & \\
\hline Bajo o muy bajo nivel de participación social & 327 & 370 & 697 & $81 \%$ & \\
\hline
\end{tabular}

Fuente: Elaboración propia a partir de los datos recogidos en "Adolescencia en riesgo social. Un estudio de las situaciones de conflicto social severo y prolongado en la adolescencia, abordadas desde el Sistema de Protección a la Infancia en Navarra” Gurbindo, M. (2014). 


\section{Gráfico 1. Distribución de menores según edad y ciclo de impacto indicadores disfuncionales} Datos absolutos. $\mathrm{N}=858$

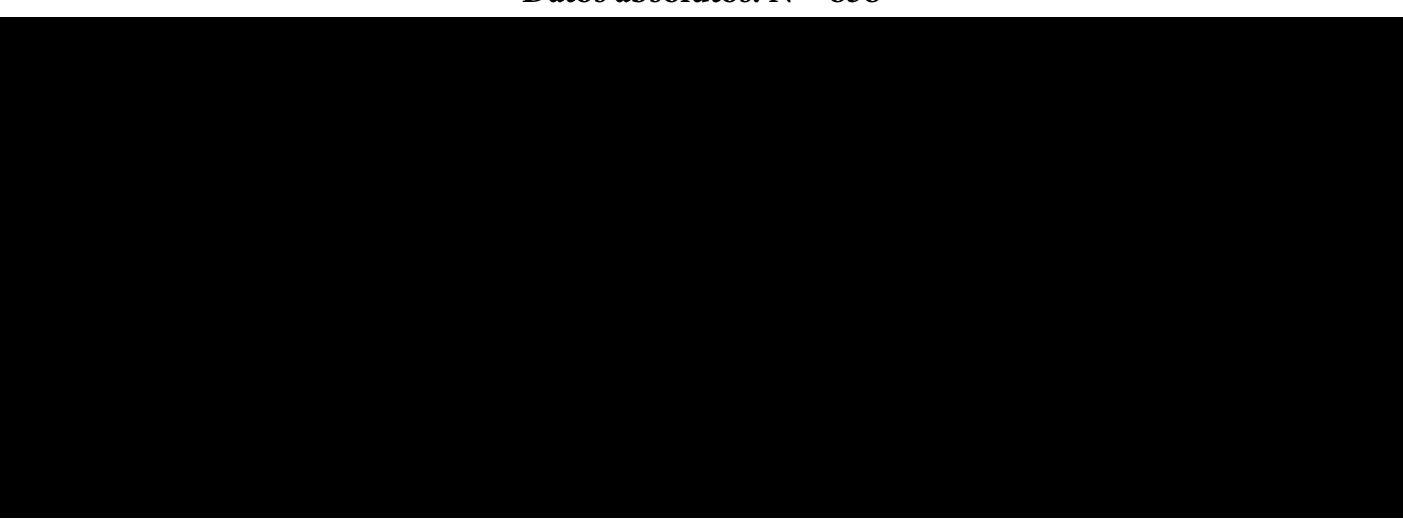

Fuente: Elaboración propia a partir de los datos recogidos en "Adolescencia en riesgo social. Un estudio de las situaciones de conflicto social severo y prolongado en la adolescencia, abordadas desde el Sistema de Protección a la Infancia en Navarra” Gurbindo, M. (2014).

de indicadores de disfuncionalidad. En este sentido, la desconfianza hacia el mundo del adulto, las dificultades en el establecimiento de relaciones interpersonales de calidad, así como la vinculación con su ámbito comunitario de referencia aparecen como los síntomas de mayor impacto, con una presencia en el $80 \%$ de los/as menores.

En cuanto al sentimiento percibido de desvalorización y culpa, así como las dificultades para vincular aparecen igualmente con un alto grado de incidencia, con una presencia en el 75 $\%$ de los/as menores. En cuanto a la variabilidad en función de la procedencia, apenas si hay diferencias significativas, apareciendo un ligero despunte por parte de la población de origen extranjero. Dentro de esta, la de origen latino, es la que más afectación presenta, debido, fundamentalmente, a los largos periodos de separación respecto de sus progenitores. Por último y en relación a la edad (ver Gráfico 1), aparecen datos significativos en cuanto al ciclo evolutivo de cada uno de los indicadores analizados. Así, podemos establecer, más allá de las diferencias propias de cada una de las categorías, que todas ellas mantienen una misma secuencia de impacto en relación al tramo de edad, de tal manera que los niveles de presencia más bajos se dan con anterioridad al inicio del periodo adolescente. El intervalo 12-14 años aparece como un espacio temporal de transición en el que la gran mayoría de indicadores aumenta progresivamente, hasta alcanzar el punto máximo de impacto en el tramo 14-16 años. Posteriormente y a lo largo del intervalo 16-18 años, se produce otro periodo temporal de transición en el que decrece la presencia e intensidad de los indicadores, hasta llegar a su punto mínimo de representación en la línea de la mayoría de edad.

De los datos obtenidos podemos intuir que la cercanía de la mayoría de edad se acaba convirtiendo en un factor de protección. En este sentido, y basándonos en la evolución llevada 
a cabo por los/as menores a lo largo del periodo de investigación, hemos podido comprobar cómo con el inicio de la crisis identitaria eclosionan las consecuencias de un vínculo y relación de apego disfuncional, apareciendo modos de comportamiento relacionales y un manejo de las emociones altamente inadaptado. No obstante y de acuerdo con otros estudios, así como por una parte importante de la literatura sobre psicología de la adolescencia, conforme avanza la edad y termina el ciclo adolescente (18-19 años), van apareciendo nuevos indicios de cambio que nos hace pensar que, junto a un mayor desarrollo cognitivo, se va desarrollando un mejor manejo y claridad de las emociones.

\section{Estilos de afrontamiento personal}

El grado que se tenga de conciencia del problema y motivación para el cambio, constituye la base para el mejor o peor establecimiento de un estilo de afrontamiento de carácter normalizado y funcional para con los sistemas del ambiente. Así, la capacidad de introspección, el modelo de atribución y motivación (externa, interna), el grado de asunción de responsabilidades, el modelo de comunicación, así como la capacidad para pedir ayuda, constituyen los indicadores sobre los que hemos basado nuestro análisis. En este sentido, y tomando como referencia los datos recogidos en la Tabla 4, destacamos, en primer lugar, la falta de menores con un nivel alto de conciencia del problema y motivación para el cambio. Igualmente es de destacar cómo el $89 \%$ de todos ellos/as presenta un bajo o muy bajo nivel de conciencia del problema y motivación para el cambio. Es de destacar cómo únicamente aparece un $11 \%$ de los/as menores con un nivel adecuado para su edad y condición de menor. De los datos obtenidos, la procedencia no establece diferencias de carácter significativo que nos haga pensar en alguna asociación diferenciada en función del origen.

En cuanto al género, la población de signo femenino presenta mejores niveles de conciencia del problema y motivación para el cambio ${ }^{11}$

11. Nivel alto: el/la menor tiene capacidad de introspección, es capaz de reconocer e identificar sus dificultades, la génesis y secuencia en que se desarrollan, así como el peso y la incidencia en su carácter y respuesta conductual. Establece mecanismos de atribución interna y asume tanto la responsabilidad de sus actos como las consecuencias que de los mismos se pudieran generar. Es capaz de verbalizar sus carencias y solicitar ayuda para el proceso de afrontamiento. Acepta y se implica de forma activa en los procesos de ayuda que se le ofrecen, acudiendo a entrevistas y encuentros, así como participando en todas aquellas actividades y/o programas que desde el Sistema de Protección se le plantean. Nivel medio: el/la menor tiene dificultades para establecer procesos de reflexión interna, reconoce la presencia de dificultades aunque no es capaz de establecer la secuencia de su génesis y desarrollo, así como del peso e incidencia en su respuesta conductual. Tiende a establecer mecanismos de atribución externa, no asumiendo enteramente la responsabilidad de sus actos ni las consecuencias que de ellos se derivan. Le cuesta admitir sus carencias, así como solicitar ayuda profesional para el afrontamiento de las dificultades. Acepta los procesos de ayuda que se le ofertan pero mantiene un nivel de implicación pasivo, presentando muchos altibajos e incidencias en su nivel de participación a entrevistas, encuentros u otros programas, así como actividades que desde las diversas instancias del Sistema de Protección se le pudieran ofertar.

Nivel bajo: el/la menor no es capaz de establecer procesos de introspección y/o reflexión sobre sus dificultades. No es capaz de establecer la secuencia en cuanto a la génesis, desarrollo e incidencia de las dificultades en su patrón conductual. Establece mecanismos de atribución externa, aunque llega a asumir cierta responsabilidad en sus actos, pero no las consecuencias que de los mismos se pudieran derivar. Se muestra con mucha ambivalencia a la hora de solicitar ayuda, e incoherente a la hora de afrontar y participar en los procesos de ayuda, produciéndose muchos altibajos e incidencias, siendo necesario ponerles fin, dada la inoperancia e imposibilidad de continuidad de los mismos.

Muy bajo: el/la menor se niega a establecer cualquier proceso de reflexión interna que suponga un reconocimiento de la situación problema. Niega de forma expresa la existencia 
Tabla 4. Distribución de menores según origen nivel de conciencia del problema y motivación para el cambio

\begin{tabular}{|c|c|c|c|c|c|c|}
\hline \multirow{2}{*}{$\begin{array}{c}\mathrm{N}=858 \text { Conciencia del problema y } \\
\text { motivación para el cambio }\end{array}$} & \multicolumn{2}{|c|}{ Menores de origen extranjero } & \multicolumn{2}{|c|}{ Menores de origen nativo } & \multirow[t]{2}{*}{$\mathrm{N}$} & \multirow[t]{2}{*}{$\%$} \\
\hline & $\mathrm{N}$ & $\%$ & $\mathrm{~N}$ & $\%$ & & \\
\hline N. Alto & 0 & & 0 & & 0 & \\
\hline N. Medio & 59 & $13 \%$ & 36 & $9 \%$ & 95 & $11 \%$ \\
\hline N. Bajo & 178 & $40 \%$ & 195 & $47 \%$ & 373 & $43 \%$ \\
\hline N. Muy bajo & 210 & $47 \%$ & 180 & $44 \%$ & 390 & $46 \%$ \\
\hline TOTAL & 447 & $100 \%$ & 411 & $100 \%$ & 858 & $100 \%$ \\
\hline
\end{tabular}

Fuente: Elaboración propia a partir de los datos recogidos en "Adolescencia en riesgo social. Un estudio de las situaciones de conflicto social severo y prolongado en la adolescencia, abordadas desde el Sistema de Protección a la Infancia en Navarra” Gurbindo, M. (2014).

Gráfico 2. Distribución de menores según media de edad y grado de conciencia del problema y motivación para el cambio

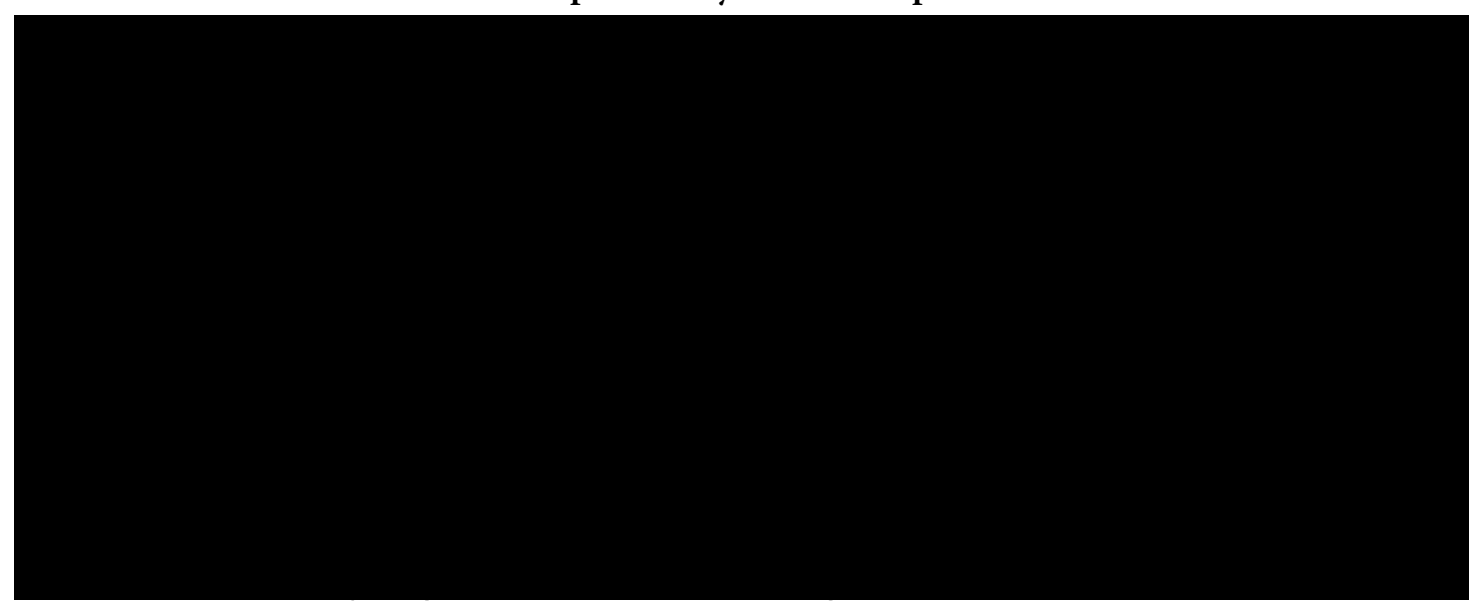

Fuente: Elaboración propia a partir de los datos recogidos en "Adolescencia en riesgo social. Un estudio de las situaciones de conflicto social severo y prolongado en la adolescencia, abordadas desde el Sistema de Protección a la Infancia en Navarra" Gurbindo, M. (2014).

En general, presentan una mejor capacidad de introspección, adquiriendo con el tiempo mejores competencias, habilidades y estrategias

del mismo, y cuando la reconoce, la sitúa enteramente en el espacio de terceras personas. Establece de forma continua mecanismos de atribución externa, negando cualquier responsabilidad personal en la génesis y desarrollo de las dificultades, así como de las consecuencias que de sus actos se pudieran derivar. En cuanto al afrontamiento de los problemas, se opone de forma expresa a participar en ningún programa de intervención, siendo únicamente posible intervenir por vía de oficio. para solicitar ayuda, así como para desarrollar mejores niveles de motivación interna que los menores de signo masculino. Por último, es de destacar la correlación positiva que se da entre el aumento de la edad y la adquisición de mejores niveles de conciencia del problema. En este sentido, el Gráfico 2 refleja la curva que se establece cuando ponemos en relación la edad con el grado de identificación, conocimiento y gestión de las emociones; de tal manera, que 
vuelve a establecerse una tendencia de mejora en la calidad de las interacciones conforme aumenta la media de edad. En nuestro caso, esta tendencia estaría en parte justificada, además por el aumento de la edad, por los procesos de supervisión, ayuda y acompañamiento llevados a cabo con el/la menor a lo largo del proceso de intervención. La percepción de verse reconocido y aceptado desde la incondicionalidad y la cercanía afectiva, posibilita, de igual manera, un aumento de la autoestima y la autoconfianza; elementos estos, esenciales para el desarrollo de la inteligencia emocional.

Una gran mayoría de los/as menores objeto de atención desarrolla un estilo de afrontamiento personal establecido en dos fases diferenciadas en el tiempo. En una primera fase, desde modelos de atribución externa, tendiendo a minimizar las dificultades, y en todo caso, responsabilizar de sus actos a terceras perso- nas (fundamentalmente a las figuras progenitoras). En este contexto, como hemos podido ver anteriormente, apenas si hay conciencia del problema y, mucho menos, implicación para el cambio. El/la menor actúa a partir de estímulos externos (dinero y objetos de consumo, experiencias nuevas, consumos, reconocimiento y señas de identidad de su grupo de iguales...). La respuesta conductual está transversalizada por el enfrentamiento con la estructura familiar y la identificación con el grupo de iguales. Así mismo, está revestida de una muy baja tolerancia a la frustración, una alta impulsividad, dificultades para empatizar, así como para asumir las consecuencias de sus actos.

Posteriormente, conforme avanza el tiempo de la adolescencia, en nuestra población objeto de atención, se va poniendo de manifiesto un contexto de mayor conflicto, en el que se hace inviable la convivencia en el medio familiar y

\section{Gráfico 3. Distribución de menores según presencia de indicadores de afrontamiento disfuncional} $\mathrm{N}=858$

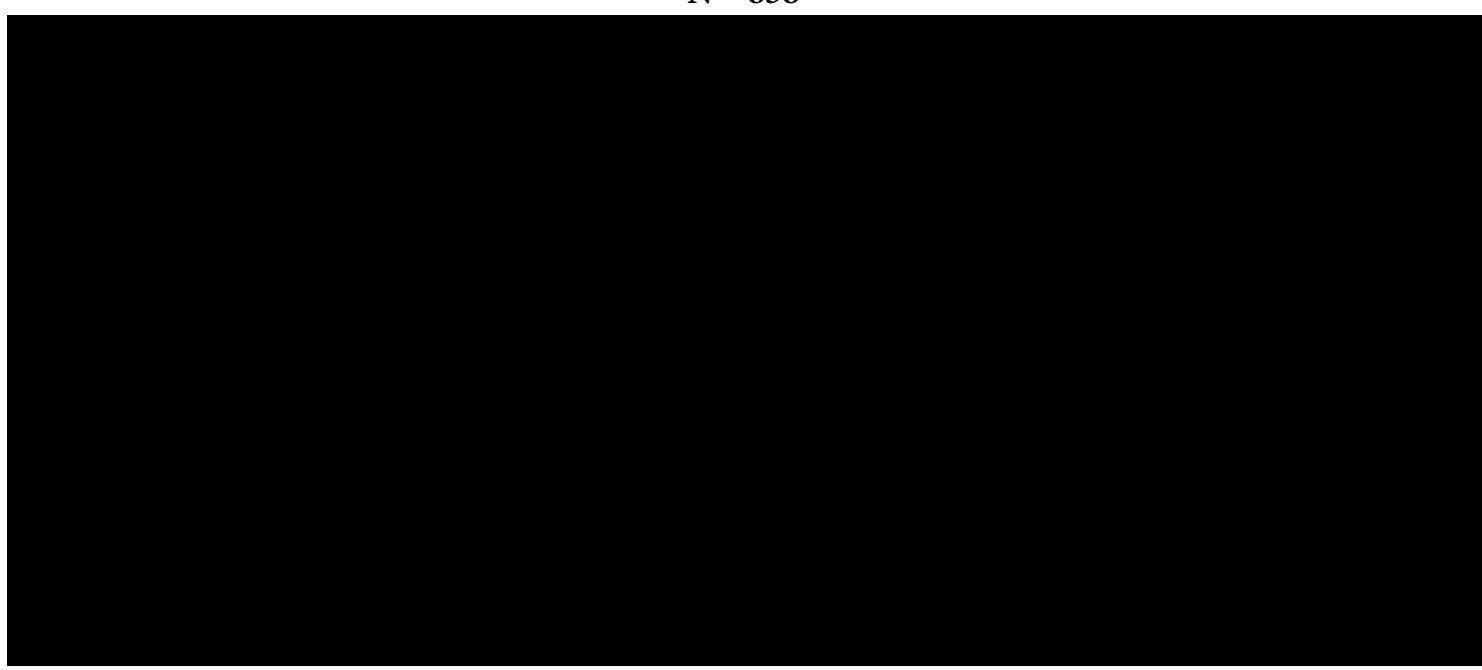

Fuente: Elaboración propia a partir de los datos recogidos en "Adolescencia en riesgo social. Un estudio de las situaciones de conflicto social severo y prolongado en la adolescencia, abordadas desde el Sistema de Protección a la Infancia en Navarra” Gurbindo, M. (2014). 
comienza a resentirse el vínculo con su grupo de iguales. Aparecen los primeros signos de desesperanza, angustia y pérdida de confianza en sí mismo/a. En este tiempo, lo importante ya no es la búsqueda de sensaciones nuevas, de ir contra la norma, la escuela, las instituciones de control o las propias figuras parentales de referencia. Lo importante ahora consiste en no estar mal, en combatir el estado de angustia, desorientación e incertidumbre. En este tiempo, en el que se hace tan necesaria la contención emocional, el/la menor no es consciente de su bajo control sobre sus emociones, sobre los diferentes aspectos de su vida, en especial, aquellos vinculados al área familiar, personal, cognitiva y de salud mental. Los/as menores en estas circunstancias, no terminan de verse como sujeto activos de cambio, reaccionando de manera pesimista, con alta preocupación e incluso con ideas de carácter auto punitivas.

En este sentido, el Gráfico 3 muestra los indicadores de reacción y afrontamiento más prevalentes en la población objeto de estudio. Como se puede apreciar, además de ponerse de manifiesto un alto grado de linealidad y comorbilidad, en cuanto al número e incidencia en la población objeto de análisis, aparecen igualmente representados la mayor parte de los indicadores de riesgo que configuran (más allá de su génesis), la secuencia tradicional en cuanto a la evolución y desarrollo del comportamiento inadaptado. En este sentido, se puede apreciar cómo el 93 \% de los/as menores mantienen como formas mayoritarias de expresión común y compartidas: 1 . Un alto grado de im- pulsividad; 2. Baja o muy baja tolerancia a la frustración, la agresividad como respuesta inmediata; y 3. Un alto grado de individualismo, así como una elevada dificultad para establecer procesos de empatía. Detectamos además, una falta de interiorización del concepto bien-mal, así como una ausencia de habilidades sociales para la introspección. Todo lo cual, hace que estemos hablando de una población con un alto grado de necesidad en cuanto a contención emocional y acompañamiento terapéutico.

\section{Conclusiones}

El análisis de los factores e indicadores de riesgo, asociados a la estructura de personalidad en lo que se refiere al ajuste psicológico-emocional de los y las menores objeto de atención, ha constituido a lo largo de la investigación, uno de los focos prioritarios de atención. Esto es así, por entender que más allá de otros condicionantes de carácter socio-familiar, la etapa adolescente constituye un tiempo en el que se ponen de manifiesto y consolidan determinados rasgos prototípicos de personalidad. Así mismo, aparecen nuevas preocupaciones, angustias y síndromes clínicos diferenciados. Todo ello, junto a lo aportado por la historia de vida particular de cada menor (crianza, vínculo, relación de apego, transiciones vitales, experiencias estresantes no normativas...), ayudan a configurar una determinada manera de interaccionar, de manejarse en la relación con los sistemas del ambiente, etc. En definitiva, una manera de percibirse a sí mismo en la relación con los(as) demás. En este senti- 
do, hemos valorado igualmente necesario establecer el grado de relación, existente entre la conducta inadaptada como primera expresión del conflicto social adolescente y los rasgos de personalidad más prevalentes en la población objeto de nuestro análisis. De los resultados obtenidos, se pone claramente de manifiesto el alto grado de correlación existente entre la presencia de manifestaciones conductuales desadaptadas en el periodo adolescente con una infancia carente de apego seguro, unos niveles de autoestima y autovalimiento deficitarios, así como con un modelo de afrontamiento disfuncional respecto de las situaciones de crisis. Los resultados obtenidos nos indican de manera general la presencia de un continuo sentimiento de malestar personal, caracterizado por:

- Falta de vínculo con sus figuras parentales o establecido desde modelos de apego inseguro desorganizado.

- Falta de conciencia del problema y motivación para el cambio.

- Procesos cognitivos basados en la atribución externa.

- Falta de motivación interna.

- Niveles altamente deficitarios de autoestima y autovalimiento.

- Falta de competencias en habilidades de interacción social.

- Dificultad para el control de impulsos y baja tolerancia a la frustración.

- Altos niveles de dependencia en las relaciones interpersonales.

- Importantes dificultades para captar y expresar sentimientos y emociones.
- Un patrón de adaptabilidad a situaciones nuevas o estresantes, deficitario o muy deficitario.

- Desarrollo de sintomatologías asociadas a trastornos propios del ámbito de salud mental.

De todo ello hemos podido constatar la presencia de un perfil de personalidad con presencia de rasgos de carácter rebelde, rudo y oposicionista, con un alto componente histriónico, alta impulsividad y baja o muy baja tolerancia a la frustración. Aspectos todos ellos, asociados a un perfil con una alta tendencia hacia lo disocial, haciendo posible una mayor predisposición a tener conflictos en los ámbitos familiar y escolar, así como a comportamientos imprevistos que pueden cuestionar los derechos de los demás. Así mismo, se ha puesto de manifiesto una alta predisposición a la impulsividad, que, de no gestionarse de forma adecuada, puede llevar a reaccionar con irritabilidad y agresividad ante la frustración. Por último, destaca la presencia de un alto grado de la inhibición a la hora de afrontar los problemas; inhibición, en este caso, asociada al alto grado de atribución externa presente en una gran mayoría de nuestros usuarios.

La presencia de todos estos factores e indicadores de riesgo, así como la falta de recursos generados por los/as propios menores condicionan en gran medida (junto con otros de carácter social) el grado de ajuste cognitivo, personal, psicológico y emocional. Como resultado de todo ello, hemos podido igualmente 
destacar con diferentes niveles de intensidad un patrón instaurado de conducta basado en:

- La inhibición en las relaciones sociales o exageración en el ejercicio de las mismas.

- Un proceso de generalización de la conducta inadaptada hacia otros sistemas del ambiente.

- Bajo rendimiento/absentismo/abandono escolar.

- Incomunicación.

- Altos niveles de agresividad.

- Conductas autodestructivas (alto grado de consumos, conductas autolíticas, conductas sexuales muy inapropiadas y de alto riesgo).

- Fuerte enfrentamiento con sus contextos referenciales de socialización (familia y escuela).

- Enfrentamiento con las instituciones de control (policial y judicial).

Así mismo, y tomando como referencia el efecto de las variables género, procedencia $y$ edad, hemos podido poner de manifiesto matices diferenciadores. De tal manera, que la procedencia (el origen de los(as) menores atendidos), aparece en todas las aproximaciones llevadas a cabo como un claro factor de riesgo. Esto es así, en tanto que en la población de origen extranjero, los indicadores analizados aparecen con una mayor intensidad. La explicación reside, en las consecuencias derivadas de la secuencia del proyecto migratorio, basadas en largos periodos de separación, aumento de la conflictiva familiar y desestabilización conyugal, un mayor repunte de la monoparentalidad sobrevenida, conflicto de lealtades de carácter cultural, así como una mayor concentración de indicadores asociados al espacio social de la exclusión. Todo lo cual, ha ido propiciando un debilitamiento de la relación vincular y de apego entre los/as menores y sus progenitores, un debilitamiento de los subsistemas parental y jerárquico, mayores niveles de desacuerdo paterno-filiar, así como un distanciamiento entre sus miembros.

Con respecto a la variable género, la población femenina sobresale con cierta claridad en las preocupaciones asociadas a los niveles de autoestima y autoconfianza, a la autoimagen personal, la configuración de la identidad y la autoevaluación negativa hacia el propio cuerpo. Todo lo cual nos apunta hacia dinámicas conductuales como expresión del alto malestar interno claramente diferenciadas. De esta manera, la población de signo femenino despunta en los síndromes asociados a comportamientos con una mayor composición interna: trastornos y sintomatología del estado de ánimo asociados al vínculo, así como a las conductas de carácter autopunitivas. Por parte de la población masculina, aparece con una mayor prevalencia en un rango de síndromes asociados a conductas con un alto grado externalizante: abuso de sustancias, alta impulsividad y tendencia a comportamientos de carácter delictivo.

Por último y en relación a la edad, se confirma la correspondencia establecida entre el tramo final de la adolescencia y un descenso en la intensidad, grado de generalización y 
comorbilidad de la conducta desadaptada. En nuestro caso, y como hemos podido constatar, el tramo 16-19 años constituye un escenario temporal en el que paulatinamente va decreciendo el grado de intensidad de los factores e indicadores disfuncionales en el ajuste psicológico-emocional, mejorando en el desarrollo de los niveles de resiliencia personal y familiar, estrategias cognitivas para afrontamiento de eventos o situaciones estresante, así como en la capacidad para pedir ayuda.

\section{Referencias}

Ainsworth, M. \& Bell, S. (1970). Apego, exploración y separación, ilustrados a través dela conducta de niños de un año en una situación extraña. En J. Delval (Comp.), Lecturasde psicología del niño, Vol. 1. Madrid: Alianza.

Aguirre, A. (1994). Psicología de la adolescencia. Barcelona. Marcombo.

Barudi, J. \& Dantagnan, M. (2005). Los buenos tratos a la infancia. Parentalidad, apego y resiliencia. Barclona: Gedisa.

Bowlby, J. (1969). El vínculo afectivo. Buenos Aires: Paidós.

Bruner, J. (1988). Realidad mental y mundos posibles. Los actos de la imaginación que dan sentido a la experiencia. Traducción de Beatriz López. Barcelona: Gedisa.

Escudero, V. (2013). Guía práctica para la intervención familiar II. Contextos familiares cronificados o de especial dificultad Junta de Castilla y León, 2013 Consejería de Familia e Igualdad de Oportunidades Gerencia de Servicios Social.
Fulquez Castro, S. C. (2010). La inteligencia emocional y el ajuste psicológico: un estudio transcultural. Tesis Doctoral. Departamento de Psicología Básica Facultat de Psicologia, Ciències de l'Educació i de l'Esport Blanquerna. Universitat Ramon Llull.

García Mediavilla, L. (1989). Test MRA. Modos de Reacción y Adaptación: personal, familiar, escolar y social. Madrid: Bruño.

Gobierno de Navarra (2002). Manual de intervención con Menores en Situaciones de Dificultad Social. Edita Gobierno de Navarra.

Gobierno de Navarra (2005). Ley Foral 15/2005, de 5 de diciembre, de Promoción, Atención y Protección a la Infancia y a la Adolescencia. Edita Gobierno de Navarra.

Gobierno de Navarra (2007). Instituto Navarro de Estadística.

Gurbindo, M. (2014). Adolescencia en riesgo social. Un estudio de las situaciones de conflicto social severo y prolongado en la adolescencia, abordadas desde el Sistema de Protección a la Infancia en Navarra. Tesis Doctoral. UPNA. Navarra.

Millon, T. (1999). Inventario Clínico Multiaxial de Millon. Pearson Clinical \& Talent Assessment. Madrid.

Stake, R. (1995). Investigación con estudio de casos. Madrid: Morata. 